\title{
Comparación entre Dos Métodos Utilizados para Medir la Curva Lumbar
}

\author{
Comparison of Two Method for Measuring the Lumbar Curve
}

"Yuing, F. T. A.; "Almagià, A. F.; "Lizana, P. J.; "Rodríguez, R. F. J.; "'Ivanovic, D. M.; **** Binvignat, G. O.; ${ }^{* * * *}$ Gallardo, L. R.; ${ }^{* * * * *}$ Nieto, C. F. \& ${ }^{* * * *}$ Verdejo, S. A.

\begin{abstract}
YUING, F. T. A.; ALMAGIÀ, A. F.; LIZANA, P. J.; RODRIGUEZ, R. F. J.; IVANOVIC, D.; BINVIGNAT, G. O.; GALLARDO, L. R.; NIETO, C. F. \& VERDEJO, S. A. Comparación entre dos métodos utilizados para medir la curva lumbar. Int. J. Morphol., 28(2):509-513, 2010.
\end{abstract}

RESUMEN: Las alteraciones posturales tales como hiperlordosis, aumento de la cifosis, cifolordosis y escoliosis en columna, producen complicaciones a nivel músculo-esquelético y articular. Debido a esto, es que en el último tiempo ha aumentado el número de atenciones kinésicas por patologías de la columna lumbar, he aquí la importancia de un test clínico que cuente con la confiabilidad necesaria para determinar la curvatura lumbar. El objetivo de este estudio es determinar si existen diferencias en los resultados entre los dos métodos utilizados para medir la curva lumbar: el test clínico de las flechas sagitales y el ángulo de la columna lumbar mediante radiografías. En una muestra de 32 pacientes de edad promedio 44,93 (+/- 12,67 años) evaluados en el Servicio de Kinesiología del Centro Médico Megasalud de Viña del Mar entre los meses de Marzo y Agosto del 2008. El procedimiento utilizado fue el examen clínico, en el cual se usó una plomada que va desde C7, pasando por la línea ínterglútea. En el plano sagital se mide la curvatura lumbar a nivel de L3. Para la medición del ángulo de la curvatura lumbar se utilizó una radiografía proyección lateral en la cual se proyectan las mesetas superior de L1 e inferior de L5 para la conformación del ángulo lumbar y su posterior medición. El resultado obtenido por medio del examen clínico entre el test de las flechas sagitales y las radiografías del ángulo de la lordosis lumbar para un índice de concordancia de Kappa, fue mayor o igual a un $95 \%$. De esta manera, se obtuvo que el test de las flechas sagitales es una herramienta válida para la pesquisa de patologías lumbares, al igual que la radiografía, con una marcada diferencia en el costo económico entre ambas.

PALABRAS CLAVE: Test de las flechas sagitales; Ángulo de la columna lumbar; Curva lumbar.

\section{INTRODUCCIÓN}

La curva lumbar es más pronunciada en las mujeres que en los hombres, comienza en la mitad de la duodécima vértebra dorsal y termina en el ángulo sacro vertebral. Dicho segmento aloja la parte final de la médula espinal y a la cauda equina, desde donde se proyectan los 5 pares de nervios espinales lumbares por los forámenes de conjunción hacia músculos, ligamentos y la piel de la región inguinal, lumbar y de los miembros inferiores. La conformación ósea del segmento lumbar genera el ángulo lumbosacro que se forma entre el plano horizontal y el plano inclinado de la superficie superior del sacro en el plano lateral. La quinta vértebra lumbar, y por consiguiente el resto de la columna vertebral que se encuentra por superior del plano inclinado, ejerce una fuerza deslizante cizallante en sentido anteroinferior (Kapandji, 2002). Cuando este ángulo crece el declive del plano también aumenta, provocando dolor por distensión de estructuras ligamentosas y sobrecarga de estructuras articulares. Además, en posición estática, el aumento del ángulo condiciona una hiperlordosis que es causa de dolor por varios mecanismos: compresión del disco intervertebral lumbosacro en su parte posterior, sobrecarga en las articulaciones interapofisiarias, estrechamiento del foramen de conjunción lumbosacro y compresión radicular (Adams \& Hutton,

* Laboratorio de Antropología Física y Anatomía Humana, Instituto de Biología, Facultad de Ciencias, Pontificia Universidad Católica de Valparaíso. Chile.

** Instituto de Nutrición y Tecnología de los Alimentos, Universidad de Chile, Chile.

*** Centro Anatómico, Campus Talca, Universidad Autónoma. Chile; Profesor colaborador Laboratorio de Antropología Física y Anatomía Humana, Instituto de Biología, Facultad de Ciencias, Pontificia Universidad Católica de Valparaíso, Chile.

***** Escuela de Kinesiología, Universidad del Mar, Chile. 
1985). Además del ángulo lumbosacro existen otros ángulos lumbares que determinan la curvatura lumbar a los cuales no se les debería restar importancia, como es el caso del ángulo de la lordosis lumbar, el cual se observa en una radiografía de proyección lateral por la intersección de dos rectas: una que va desde la parte superior de la primera vértebra lumbar y la otra de la meseta superior del sacro. Este ángulo puede estar influido por el ángulo lumbosacro y es de importancia a la hora de una evaluación de la curvatura lumbar (Chernukha et al., 1998).

Dadas las consecuencias expresadas en los párrafos previos sobre las posibles consecuencias de un aumento de la curva lumbar es que ha sido ampliamente utilizada la radiografía de proyección lateral para evaluar la conformación del segmento lumbar, dejando de lado el examen físico y la realización de pruebas clínicas. Consistente con esto, es que el propósito principal de esta investigación es determinar el grado de correlación entre el test de flechas sagitales y el ángulo de lordosis lumbar en una radiografía de proyección lateral.

\section{SUJETOS Y MÉTODO}

Se estudiaron 32 sujetos seleccionados de una muestra intencionada de un universo de 360 de ambos sexos, 17 hombres $(53,12 \%)$ y 15 mujeres $(46,87 \%)$ de edad promedio 46,97 (+/-12,69) años, todos correspondientes a pacientes del Servicio de Kinesiología del Centro Médico
Megasalud de Viña del Mar, Chile, con diagnóstico de patología lumbar, que presentaran radiografía de proyección lateral al momento de la evaluación y que cumplieran con los criterios de inclusión. Las variables evaluadas fueron: la medición de la lordosis lumbar a través del test de las flechas sagitales mediante el protocolo propuesto por Santoja \& Martínez (1992) (Fig. 1) y el ángulo de lordosis lumbar en una radiografía de proyección lateral mediante el protocolo de Tribastone (1998).

La evaluación de la curva lumbar mediante el test de flechas sagitales se realizó mediante la determinación de la distancia existente entre la vertical de la plomada hasta el punto más alejado de la misma y que clasifica los valores como hiperlordosis si el valor es mayor a $35 \mathrm{~mm}$, normal entre 20 y $35 \mathrm{~mm}$ e hipolordosis si el valor es menor a 20 $\mathrm{mm}$. Para la medición del ángulo de lordosis en una radiografía se realizó la proyección de la meseta superior de L1 e inferior de L5 para formar y determinar el ángulo lumbar y que clasifica los valores como hiperlordosis si el valor es mayor a $45^{\circ}$, normal entre $25^{\circ}$ y $45^{\circ}$ e hipolordosis si el valor es menor a $25^{\circ}$. Las evaluaciones fueron realizadas previo consentimiento informado de los sujetos, en las mismas condiciones de hora de evaluación, espacio y temperatura durante el proceso de recolección de datos.

Para el análisis y tratamiento estadístico se utilizó el software Statistic 6.0, para la estadística paramétrica de tendencia central y para el análisis inferencial se utilizó la proporción de concordancia de Kappa, con un nivel de significancia del $1 \%$.

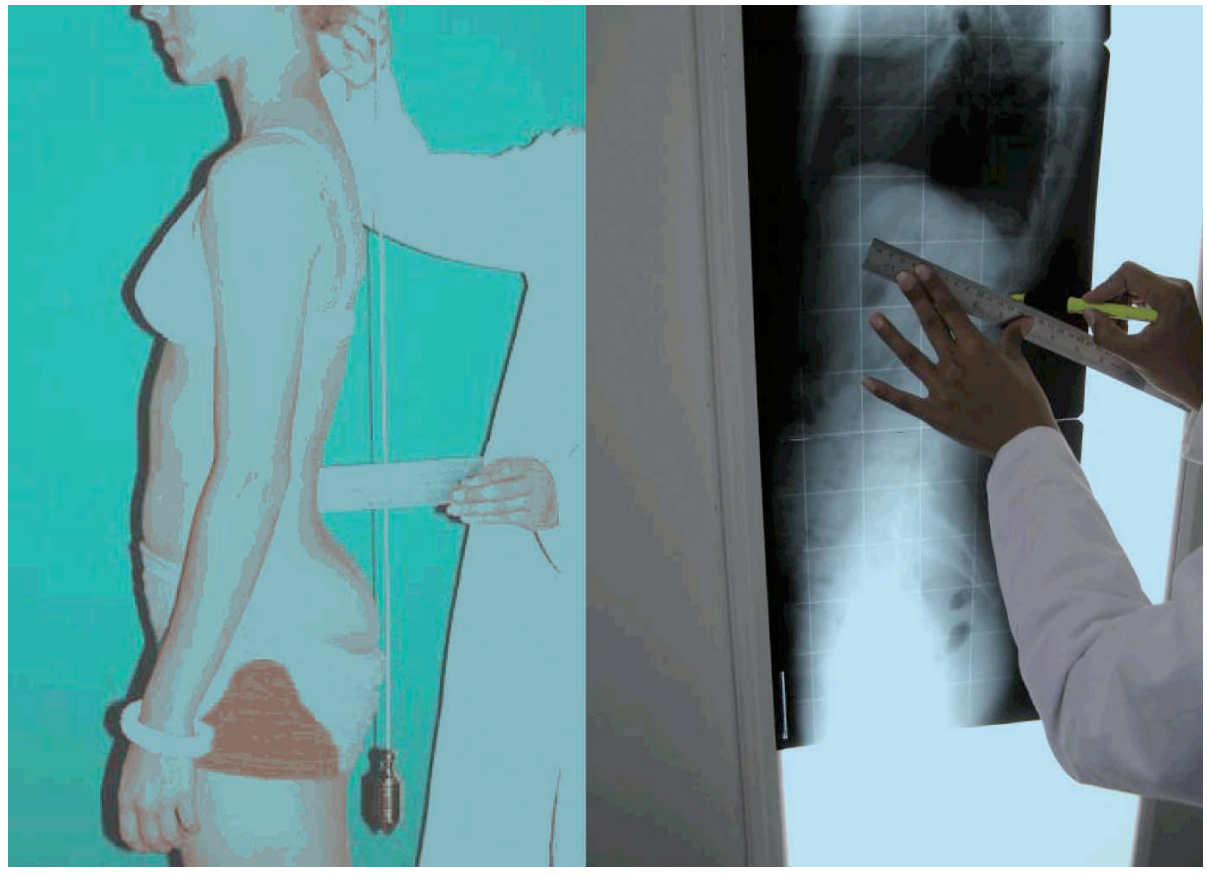

Fig. 1. Medición de la Curva Lumbar mediante el Test de Flechas Sagitales (a la izquierda) y Medición del Ángulo de Lordosis Lumbar Radiográfico (a la derecha). 


\section{RESULTADOS}

En la Tabla I, se aprecian los resultados de la evaluación de la curva lumbar mediante el test de flechas sagitales, donde se observa que el 90,6\% de los sujetos se clasifican dentro de la categoría de hiperlordosis. En la Tabla II se consideran los resultados de las mediciones de la curva lumbar mediante el ángulo de lordosis radiográfico, el 78,1 \% de los sujetos se clasifica en la categoría de hiperlordosis. Relacionando las variables se obtuvo que el 87,5 \% de los sujetos coincidieron con su clasificación diagnóstica, independiente del método utilizado (Tabla III). De la evaluación de la curva lumbar mediante el test de flechas sagitales, del 90,6\% de los sujetos clasificados dentro de la categoría de hiperlordosis el $46,9 \%$ corresponde a hombres y el restante $43,8 \%$ a mujeres. Además sólo el 6,3\% de los sujetos se clasificó dentro de la categoría normal. En relación a la evaluación de la curva lumbar mediante radiografía, del 78,1\% de los sujetos clasificados dentro de la categoría de hiperlordosis el $40,6 \%$ corresponde a hombres y el restante 37,5\% a mujeres y sólo el 18,8\% de los sujetos se clasificó dentro de la categoría normal (Tablas IV y V respectivamente). Mediante el test de hipótesis de concordancia, se determinó un 0,875 con un índice Kappa que según los criterios de valoración del índice avalarían que la concordancia entre las variables es muy buena o muy alta, existiendo un alto grado de asociación entre las clasificaciones establecidas mediante flechas sagitales y las radiografías.

En la Tabla V se muestra la frecuencia alcanzada con ambos métodos de medición, en la cual 25 pacientes correspondientes al 78,1\% del total de 32 de la muestra obtuvieron la clasificación de hiperlordosis, pero un $12,5 \%$ de los pacientes en estudio no ratifica lo indicado por la radiografía. De una muestra de 32 pacientes, 4 arrojaron resultados diferentes entre el test de las flechas sagitales y el ángulo de la lordosis lumbar usando radiografía, 3 de éstos presentan diferencias en la clasificación y sólo uno de ellos se encontró en el límite de corte de la medición radiográfica, con un valor de $45^{\circ}$, razón por la cual éste podría clasificarse también como hiperlordosis y ajustarse al diagnóstico del test de las flechas sagitales.

Tabla I. Categorización de los sujetos según el Test de Flechas Sagitales.

\begin{tabular}{lcc}
\hline Curva lumbar - Test de Flechas Sagitales & Frecuencia Absoluta & Frecuencia Relativa \\
\hline Hipolordosis & 1 & 3,1 \\
Normal & 2 & 6,3 \\
Hiperlordosis & 29 & 90,6 \\
Total & 32 & 100,0 \\
\hline
\end{tabular}

Tabla II. Categorización de los sujetos según el ángulo de lordosis lumbar radiográfico.

\begin{tabular}{lcc} 
Curva lumbar - Ángulo de Lordosis Lumbar Radiográfico & Frecuencia Absoluta & Frecuencia Relativa \\
\hline Hipolordosis & 1 & 3,1 \\
Normal & 6 & 18,8 \\
Hiperlordosis & 25 & 78,1 \\
Total & 32 & 100,0 \\
\hline
\end{tabular}

Tabla III. Correlación de la clasificación diagnóstica por ambos métodos.

\begin{tabular}{lcc}
\hline Correlación entre ambos métodos & Frecuencia Absoluta & Frecuencia Relativa \\
\hline Positivo & 28 & 87,5 \\
Negativo & 4 & 12,5 \\
Total & 32 & 100 \\
\hline
\end{tabular}

Tabla IV. Clasificación mediante el test de las flechas sagitales de la curva lumbar por sexo.

\begin{tabular}{lcccccccc}
\hline & \multicolumn{3}{c}{ Curva Lumbar } & \multicolumn{3}{c}{ Test de Flechas Sagitales } \\
\hline Sexo & \multicolumn{2}{c}{ Hipolordosis } & \multicolumn{2}{c}{ Normal } & \multicolumn{2}{c}{ Hiperlordosis } & \multicolumn{2}{c}{ Total } \\
\cline { 2 - 9 } & Frec. & $\%$ & Frec. & $\%$ & Frec. & $\%$ & Frec. & $\%$ \\
\hline Femenino & 0 & 0 & 1 & 3,1 & 14 & $\mathbf{4 3 , 8}$ & 15 & 46,9 \\
Masculino & 1 & 3,1 & 1 & 3,1 & 15 & $\mathbf{4 6 , 9}$ & 17 & 53,1 \\
Total & 1 & 3,1 & 2 & $\mathbf{6 , 2}$ & 29 & 90,6 & 32 & 100 \\
\hline
\end{tabular}


YUING, F. T. A.; ALMAGIÀ, A. F.; LIZANA, P. J.; RODRIGUEZ, R. F. J.; IVANOVIC, D.; BINVIGNAT, G. O.; GALLARDO, L. R.; NIETO, C. F. \& VERDEJO, S. A. Comparación entre dos métodos utilizados para medir la curva lumbar. Int. J. Morphol., 28(2):509-513, 2010.

Tabla V. Clasificación mediante radiografía de la curva lumbar por sexo.

\begin{tabular}{lcccccccc}
\hline & \multicolumn{3}{c}{ Ángulo de Curva Lumbar } & \multicolumn{3}{c}{ Radiografía } \\
\hline Sexo & \multicolumn{2}{c}{ Hipolordosis } & \multicolumn{2}{c}{ Normal } & Hiperlordosis & \multicolumn{2}{c}{ Total } \\
\cline { 2 - 9 } & Frec. & $\%$ & Frec. & $\%$ & Frec. & $\%$ & Frec. & $\%$ \\
\hline Femenino & 0 & 0 & 3 & 9,4 & 12 & $\mathbf{3 7 , 5}$ & 15 & 46,9 \\
Masculino & 1 & 3,1 & 3 & 9,4 & 13 & $\mathbf{4 0 , 6}$ & 17 & 53,1 \\
Total & 1 & 3,1 & 6 & $\mathbf{1 8 , 8}$ & 25 & 78,1 & 32 & 100 \\
\hline
\end{tabular}

\section{DISCUSIÓN}

Diagnosticar un aumento o disminución de la curva lumbar en pacientes aparentemente sanos puede ser de costos elevados y puede obligar a la utilización de una evaluación radiográfica en estos pacientes. Es por esta razón que hacer un diagnóstico precoz de los cambios anormales de la curvatura lumbar, ya sea por aumento o disminución de ésta, nos permitirá tomar las medidas necesarias para evitar las posibles patologías que se pueden producir por no contar con una curvatura equilibrada. Al igual que lo demostrado por Chernukha et al. donde se indica que en ciertas condiciones de cambio en la curva de la lordosis lumbar puede indicar o incluso preceder a la aparición de síntomas neurológicos. Es por esto que es necesaria una prueba para medir la curvatura lumbar que cuente con toda la confianza del evaluador para excluir a aquellos pacientes que presenten una curvatura lordótica normal antes de someterlos a una evaluación radiológica. Los datos obtenidos en nuestro estudio demuestran que la concordancia encontrada en la clasificación de lordosis lumbar entre ambos métodos tiene una confiabilidad del 95\%, cuyo índice Kappa es de 0,88 aproximadamente, lo cual muestra una significativa asociación o relación existente entre ambas clasificaciones, el test de las flechas sagitales versus la medición radiográfica de la curva lumbar, siendo concordante nuestros resultados con otras investigaciones (Olmedo et al., 2006).

Ante el problema ¿qué método será el indicado para medir la curvatura lumbar? La respuesta estará en manos del evaluador y de la experiencia que él tenga, pero evidencia como la obtenida puede ayudar a tener más confianza en los resultados que arrojen las mediciones realizadas por medio del test de flechas sagitales. Similares resultados obtuvo Silva (2001), quien asoció mediante la determinación de la curvatura lumbar en adolescentes la generación de patologías a nivel lumbar. A través de la medición arrojada en el test de flechas sagitales un 90,6\% de los pacientes estudiados presentaron hiperlordosis lumbar, siendo esta clasificación la más frecuentemente utilizada, lo cual se presenta en la Tabla IV. En relación con lo anterior, en la Tabla $\mathrm{V}$ un $78,1 \%$ de los pacientes que fueron clasificados me- diante radiografía padecían hiperlordosis lumbar, siendo el $3,1 \%$ clasificados con hipolordosis. Esto podría tener relación con los resultados obtenidos en el estudio de Raue et al. (1975), en donde la mayoría de los evaluados en su estudio padecían hiperlordosis lumbar. Dentro de los resultados obtenidos en la Tabla III, en la cual los pacientes fueron evaluados con ambos métodos, sin importar la clasificación en la que se ubicaran de la curvatura lumbar, existió un $87,5 \%$ de concordancia entre los test lo cual equivale a 28 pacientes que coincidieron del total de la muestra. La muestra es equilibrada entre hombres y mujeres, lo cual nos aportaría la seguridad que los métodos no difieren según el sexo del paciente, corroborándose posteriormente en la Tabla IV en la cual se demuestra que los hombres corresponden a un $53,1 \%$ y las mujeres a un $46,9 \%$ de la muestra. Nuestros datos de medición radiográfica contrastan con los datos obtenidos en la investigación de Polly et al. (1996), en la cual evaluaron la fiabilidad y reproducibilidad de la medición de la lordosis lumbar a través de la radiografía, obteniendo como resultado que la medición de la curva lumbar es reproducible y fiable si la técnica se especifica. Es importante señalar que el paciente diagnosticado con hipolordosis es el mismo para ambos test de medición.

\section{AGRADECIMIENTOS}

En primer lugar a cada una de las personas participantes de la investigación, a la Institución Megasalud por la autorización y facilidades para la realización de la misma y a cada uno del grupo de trabajo del Laboratorio de Antropología Física y Anatomía Humana de la Pontificia Universidad Católica de Valparaíso, por la constancia y colaboración en el trabajo. Además agradecer a la Dirección de Investigación, Vice-rectoría de Investigación y Estudios Avanzados e Instituto de Biología de la Facultad de Ciencias de la Pontificia Universidad Católica de Valparaíso, Chile, por su constante apoyo en materia investigativa. 
YUING, F. T. A.; ALMAGIÀ, A. F.; LIZANA, P. J.; RODRIGUEZ, R. F. J.; IVANOVIC, D.; BINVIGNAT, G. O.; GALLARDO, L. R.; NIETO, C. F. \& VERDEJO, S. A. Comparison of two method for measuring the lumbar curve. Int. J. Morphol., 28(2):509-513, 2010.

SUMMARY: The altered posture, such as hiperlordosis, increase in kyphosis, and scoliosis in cifolordosis column complications occur at the muscular, skeletal and articulate. Because of this, is that in recent times to increase the number of benefits for kinesics pathologies of the lumbar spine, and here the importance of a clinical test that has the confidence necessary to determine lumbar curvature. The objective of the study seeks to determine whether there are differences in outcomes between the two methods used to measure the lumbar curve, the test of the arrows sagitales and the angle of the lumbar spine using X-rays in patients greater than or equal to 21 years, as assessed the service kinesiology of Centro Médico Megasalud of Viña del Mar, between the months of March and August 2008. The procedure used was by means of physical examination, which uses a plumb line that runs from $\mathrm{C} 7$ through inter gluteal line in the sagittal plane was measured at the lumbar curvature of L3 and compared with the measurement of the angle curvature of the lumbar measured by radiographic position in bipedal in a side view in 32 adults, measures of kinesiology at the service of the Medical Center Megasalud of Viña del Mar, Chile. The result obtained by means of physical examination between the test of the arrows sagitales versus the angle of the lumbar lordosis measured by X-rays, reached a record of correlation between the two diagnostic tests greater than or equal to $95 \%$. In conclusion we can say that the test of the arrows sagitales is a valid tool for the investigation of lumbar pathologies, like radiography, with a marked difference in cost between the two.

KEY WORDS: Test of the arrows sagitales; Angle of the lumbar spine; Lumbar curve.

\section{REFERENCIAS BIBLIOGRÁFICAS}

Adams, M. A. \& Hutton, W. C. The effect of posture on the lumbar spine. J. Bone Joint Surg. Br., 67(4):625-9, 1985.

Chernukha, K. V.; Daffner, R. H. \& Reigel, D. H. Lumbar lordosis measurement. A new method versus Cobb technique. Spine, 23(1):74-9, 1998.

Kapandji, A. Fisiología articular. Tronco y raquis. Madrid, Médica Panamericana, 2002. Tomo III.

Olmedo, B.; Enrique, C.; Díaz, V.; Trujillo, B. \& Millán, R. Evaluación de un marco de referencia postural como prueba diagnóstica de postura lordótica lumbar. Gac. Méd. Méx., 142(1):39-42, 2006.

Polly, D. W. Jr.; Kilkelly, F. X.; McHale, K. A.; Asplund, L. M.; Mulligan, M. \& Chang, A. S. Measurement of lumbar lordosis. Evaluation of intraobserver, interobserver, and technique variability. Spine, 21(13):1530-5, 1996.

Raue, V. I.; Sauer, I.; Jahn, K. \& Ries, W. Influence of overweight on the load-capacity of the supporting and locomotion system in women. Z. Gesamte Inn. Med., 30(15):494-8, 1975.

Santonja, F. \& Martínez, I. Valoración Médico-Deportiva del Escolar. Murcia, Universidad de Murcia, 1992.

Silva, H. Ángulos del plano sagital de la columna lumbosacra en una muestra de adolescentes de la ciudad de Temuco, Chile. Rev. Chil. Anat., 19(3):271-7, 2001.
Tribastone, F. Compendio de gimnasia correctiva, $3^{\mathrm{a}}$ ed. Barcelona, Paidotribo, 1998.

Dirección para correspondencia:

Prof. Atilio Aldo Almagiá Flores

Avda. Brasil 2950 Casilla 4059

Pontificia Universidad Católica de Valparaíso

Valparaíso

CHILE

Email: aalmagia@ucv.cl

Recibido: 25-02-2010

Aceptado: 22-03-2010 
